\title{
Los estilos de liderazgo y la gestión de recursos humanos: una reflexión acerca del impacto sobre el desempeño de los estudiantes en la educación media
}

\author{
Leadership styles and human resources management: \\ theoretical reflection about the impact on the secondary school \\ student results
}

\author{
Liliana Pedraja-Rejas $^{1} \quad$ Emilio Rodríguez-Ponce ${ }^{2} \quad$ Carlos Villarroel González $^{3}$ \\ Recibido 9 de enero de 2012, aceptado 25 de septiembre de 2012 \\ Received: January 9, 2012 Accepted: September 25, 2012
}

\begin{abstract}
RESUMEN
En este artículo se pretende dar respuesta, desde una perspectiva teórica, a la pregunta relevante asociada a si los estilos de liderazgo y la gestión de recursos humanos por parte de la alta dirección tienen algún impacto en la eficacia de los colegios de educación media en Chile.

La motivación a este trabajo viene dada por las diferencias observadas en los niveles de calidad en la educación media, lo cual se manifiesta en los resultados de las pruebas de selección universitarias, donde queda demostrado que establecimientos educacionales de enseñanza media, que poseen poblaciones escolares equivalentes, no alcanzan los mismos resultados. En efecto, a partir de una reflexión teórica basada en el análisis crítico de un marco de referencia se llega a considerar que el liderazgo y los procesos de gestión de recursos humanos inciden finalmente en la calidad de los procesos de enseñanza y en los resultados alcanzados por los alumnos.
\end{abstract}

Palabras clave: Prueba de selección universitaria, subsidios a la oferta educacional, subsidios a la demanda educacional, equipo directivo, estilos de liderazgo, gestión de recursos humanos.

\section{ABSTRACT}

This paper seeks to answer the relevant question, from a theoretical view, if the leadership styles and human resources management have an impact on the effectiveness of schools in secondary education in Chile. The motivation for this work is given by the observed differences in the levels of quality in secondary education, which is manifested in the results of university selection tests, where it is shown that secondary school educational establishments, which have equivalent school populations, do not achieve the same results. Indeed from a theoretical reflection based on critical analysis of a framework, we show that leadership and human resources management ultimately affect the teaching quality and results achieved by students.

Keywords: University selection test, subsidies to the educational supply, subsidies to the educational demand, management team, leadership styles, human resources management.

\footnotetext{
1 Escuela Universitaria de Ingeniería Industrial, Informática y de Sistemas. Universidad de Tarapacá. Arica, Chile. E-mail: lpedraja@uta.cl

2 Instituto de Alta Investigación. Universidad de Tarapacá. Arica, Chile. E-mail: erodrigu@uta.cl

3 Escuela Universitaria de Ingeniería Eléctrica-Electrónica. Universidad de Tarapacá. Arica, Chile. E-mail: cvillar@uta.cl
} 


\section{INTRODUCCIÓN}

\section{El sistema de educación chileno}

La educación tiene un papel fundamental en la sociedad del conocimiento dado que es una de las fuentes principales de la ventaja competitiva de las naciones, siendo además un determinante esencial para la movilidad social y la equidad en la distribución del ingreso, principalmente en los países en vías de desarrollo. Muchos economistas de prestigio han destacado el impacto de la educación sobre el crecimiento económico (por ejemplo [7, 45-46]) destacándose que los retornos sociales y privados de la educación son elevados para la sociedad [59-60, 18].

La educación es un bien público o semipúblico (por ejemplo [73, 31]). De hecho, un año adicional de escolaridad tiene efectos superiores al $6 \%$ sobre la productividad individual (por ejemplo $[8,46]$ ) y un impacto superior al $0,2 \%$ sobre el producto interno bruto $[17,20,9]$. Por lo tanto, la educación genera tanto beneficios privados como beneficios públicos $\mathrm{y}$, en tal sentido, tiene un impacto sobre el bienestar social y sobre el crecimiento de la economía.

No obstante lo anterior, los problemas que enfrenta la educación en el mundo, sobre todo en los países menos desarrollados, son de una magnitud significativa. En efecto, la educación suele ser deficiente si se compara lo que los estudiantes efectivamente aprenden en relación con lo que deberían aprender de acuerdo al currículo aprobado por su país o Estado [44, 32].

Chile forma parte del gran número de países en los que se produce esta situación. La diversidad en los niveles de calidad de la educación media queda en evidencia cuando se analizan los resultados de las pruebas de selección universitaria (P.S.U.). La P.S.U. está compuesta por cuatro pruebas, las que son desarrolladas, administradas, aplicadas y reportadas por el Departamento de Evaluación, Medición y Registro Educacional (DEMRE) de la Universidad de Chile. Dichas pruebas se configuran como instrumentos de evaluación educacional que miden la capacidad de razonamiento de los postulantes egresados de la enseñanza media, teniendo como medio los contenidos del Plan de Formación General de Lenguaje y Comunicación, de Matemática, de Historia y Ciencias Sociales y de Ciencias. Las primeras dos son obligatorias, mientras que las otras dos son optativas (www.demre.cl)

Ciertamente es reduccionista pensar que la calidad de la educación media se mide por los resultados de la prueba mencionada; sin embargo, estos resultados son, al menos, uno de los indicadores relevantes que pretenden medir el nivel de dominio de contenidos de la enseñanza media.

$\mathrm{Al}$ respecto, considérese que en el proceso de admisión 2010 se inscribieron en la P.S.U. 285.325 estudiantes, donde un grupo total de 251.634 postulantes rindieron las pruebas obligatorias de Lenguaje y Comunicación y Matemática, de los cuales un $32,4 \%$ de los jóvenes ponderó por debajo de los 450 puntos, mientras que sólo un $3 \%$ logró más de 700 puntos. Una gran cantidad de estudiantes se ubicó en el tramo entre los 450 y 699 puntos $(64,5 \%)$ (Compendio Estadístico proceso de Admisión 2010, DEMRE, p. 32).

En Chile hasta inicios de los años 80 el sistema educacional se sustentaba en una educación financiada mediante subsidios a la oferta que permitían al Estado proveer educación en forma directa, junto con algunos actores privados. No obstante, a partir del año 1981 se comenzó a implementar una lógica de subsidio a la demanda que asigna los recursos financieros a las unidades educativas considerando la asistencia a clases del alumno.

La lógica que subyace en el modelo es que la libertad para elegir de los estudiantes y sus respectivas familias determinará qué colegios tendrán éxito y cuáles fracasarán por escasez de demanda. Así tendrán mayor subsidio aquellas escuelas y colegios que por su mayor calidad sean capaces de atraer la mayor cantidad y calidad de estudiantes. Sin embargo, en esta lógica existen algunos problemas prácticos: primero, la asimetría de información que existe en el sistema no permite a los estudiantes y sus familias tomar las mejores decisiones necesariamente; segundo, no existe una perfecta movilidad de recursos y de la demanda; tercero, los costos de la educación no se pueden estandarizar ya que el capital social, económico y cultural de la población escolar es un determinante estructural de sus posibilidades de aprendizaje; cuarto, la condición socioeconómica de los estudiantes es fundamental al comparar los resultados de distintos establecimientos 
educacionales; quinto, la asignación de recursos no considera debidamente los resultados logrados por los colegios, controlados por las condiciones sociales y económicas de los estudiantes; sexto, la libertad de elección se encuentra seriamente limitada por las capacidades económicas de las familias ya que los colegios particulares subvencionados exigen un copago que muchas familias no están en condición de solventar.

De modo similar, es imprescindible destacar que el modelo de financiamiento de la educación no considera dimensiones tan relevantes como: el esfuerzo, el tiempo y los costos asociados al proceso educativo que se incurren, según el grado de vulnerabilidad (menor capital social, económico y cultural) de la población atendida para alcanzar logros escolares. Consecuentemente, el Estado se preocupa de manera especial de la supervisión de la asistencia más que del aseguramiento de la calidad institucional de las escuelas y colegios [16].

Por otra parte, los resultados de las pruebas P.S.U. han demostrado que colegios que tienen poblaciones escolares equivalentes no necesariamente obtienen los mismos resultados, sino que más bien resultados que pueden ser significativamente diferentes. Más aún, en los diferentes tipos de establecimientos, es decir, municipalizados, subvencionados y privados, es posible encontrar experiencias exitosas y fracasos.

Entonces, si las diferencias socioeconómicas no permiten explicar, al menos completamente, las diferencias en los resultados de las pruebas de selección universitarias, entonces es necesario revisar aquellas otras variables que el modelo educativo y de financiamiento no ha considerado, es decir, aquellos factores que se relacionan con el ámbito de la dirección de las instituciones educativas y sus variables asociadas.

La pregunta relevante que emerge a partir de esta discusión es: ¿Los estilos de liderazgo y la gestión de recursos humanos por parte de la alta dirección tienen algún impacto en la eficacia de los colegios de educación media?

\section{Liderazgo y gestión de recursos humanos}

En el campo de la administración de organizaciones, existen dos posiciones teóricas contrapuestas en relación con la importancia del equipo de alta dirección en los procesos estratégicos y, por ende, sobre los resultados de las entidades. Por un lado, la ecología de las organizaciones sugiere que el equipo de alta dirección no tiene un papel determinante sobre el éxito de las organizaciones, las que más bien viven un proceso de selección natural y es, por tanto, el medio ambiente es el que determinará las condiciones de supervivencia (por ejemplo, [29, 12]). Por otro lado, Hambrick y Mason [27] sustentan una línea de investigación dominante en los últimos años, en la que argumentan que los resultados de las organizaciones están determinados parcialmente por las características del equipo directivo, ya que en un contexto de racionalidad limitada las miradas o visiones conceptuales que sustentan el accionar de las organizaciones están limitadas por el sesgo cognitivo y los valores del equipo, aspectos que influyen sobre la percepción selectiva, la interpretación de los actores relevantes y sobre la selección e implementación de la estrategia.

Varios estudios han dado soporte empírico y conceptual a este enfoque, demostrando que la diversidad (por ejemplo [58 70, 11]), el estilo de liderazgo (por ejemplo [19, 50,76, 52, 55-56]) y el tamaño del equipo de alta dirección (por ejemplo [25]) constituyen variables que pueden influir no sólo en los procesos de toma de decisiones, sino que en el éxito de las instituciones.

En los últimos años se ha ido incorporando con mayor fuerza el estilo de liderazgo como una variable que se incluye en la teoría de los escalafones superiores, dado que esta dimensión tiene efectos directos sobre los procesos de toma de decisiones y sobre los resultados que logran las organizaciones (por ejemplo $[54,53,57])$.

En efecto, algunos estudios han sugerido que el estilo de liderazgo influye sobre la productividad de los individuos. Por ejemplo, Evkall y Ryhammar [19] establecen que el liderazgo influye sobre el clima organizativo y el clima impacta sobre la creatividad y la productividad. Por su parte, Kahai y Sosik [38] hallan que el estilo de liderazgo participativo encuentra mayor colaboración entre los subordinados.

También se ha encontrado que el estilo de liderazgo tiene efectos sobre la flexibilidad, la responsabilidad, los estándares, las recompensas y el compromiso, así como sobre el clima organizativo [21]. La conducta 
de los subordinados puede verse influenciada por la motivación que genera el líder [67]. También se ha encontrado evidencia empírica que muestra que el estilo de liderazgo afecta el desempeño a través de la cultura organizacional [50].

Algunas investigaciones realizadas en Chile han probado que el estilo de liderazgo puede influir en los resultados de empresas públicas, de pequeñas empresas, de instituciones universitarias [52, 54]. De hecho, Rahman [61] sostiene que el liderazgo puede tener impacto sobre distintos tipos de organizaciones.

En Chile existe un déficit de investigaciones que hayan generado publicaciones internacionales y que vinculen los estilos de liderazgo con la gestión de recursos humanos y con la calidad o eficacia de la educación.

\section{El liderazgo en organizaciones educativas}

En el ámbito educacional, el liderazgo se refiere a la capacidad de la alta dirección para mejorar la escuela, crear un clima organizativo orientado al aprendizaje y para estimular el mejor esfuerzo $\mathrm{y}$ efectividad del equipo docente y administrativo $[6,36,75]$.

El estado del arte sobre el tema tiene, al menos, dos dimensiones cuyos resultados no muestran un paradigma dominante. En primer lugar, una dimensión relevante consiste en identificar si el impacto de los estilos de liderazgo debe estudiarse en la relación del alto directivo sobre sus subordinados o en una perspectiva de liderazgo compartido. En segundo lugar, se debe clarificar si el estilo de liderazgo es capaz de generar efectos directos sobre los resultados de los establecimientos educacionales o sus efectos pueden ser más bien indirectos y no necesariamente afectan la calidad y resultados de los establecimientos educativos.

Respecto de la primera dimensión, efectivamente, la investigación inicial sobre liderazgo en instituciones de educación se basó esencialmente en el liderazgo de la alta dirección (principal) y se procuró identificar las habilidades, comportamiento y estilo interpersonal que hacía efectivo al líder [80]. Esta línea de investigación ha encontrado que el estilo de liderazgo influye sobre el éxito de programas de cambio y sobre el mejoramiento de la calidad de la educación (por ejemplo, [26]).
Sin embargo, más recientemente ha surgido el concepto de liderazgo compartido. En este contexto, Spillane, Halverson y Diamond [71-72] consideran que las tareas del líder deben ser distribuidas en los distintos actores de las escuelas, sumando a los líderes formales e informales y a los profesores. De manera similar, Hatcher [34] plantea que en Inglaterra existen muchos ejemplos que demuestran que un liderazgo compartido más democrático que jerárquico promueve el éxito en las escuelas. En esta misma línea de trabajo, Gregory [22] estudia tres escuelas con alta vulnerabilidad social y descubre que el liderazgo tiene un papel fundamental en el éxito de estas escuelas, al dar poder a los profesores para desarrollar su labor. Por su parte, Harris [33] plantea que el liderazgo en establecimientos de educación se relaciona fundamentalmente con construir relaciones positivas y dar poder a otros para que realicen adecuadamente su tarea. Hui y Cheung [37] argumentan que el líder debe motivar la ayuda y el aprendizaje conjunto con sus subordinados para impactar sobre el aprendizaje de los estudiantes. Finalmente, Cooper [13] realiza un estudio en 11 escuelas de excelencia en North Carolina y descubre que los factores determinantes del éxito se asocian a la perspectiva de un liderazgo compartido y colaborativo.

En relación con la segunda dimensión a la que se hace referencia en el estado del arte, existen varios estudios que ponen de manifiesto la importancia directa del estilo de liderazgo sobre los resultados de los procesos educativos. Por ejemplo, se ha probado que el estilo de liderazgo influye sobre la administración y, subsecuentemente, sobre el desempeño de los estudiantes [35]. Previamente, Silins [69] había demostrado que tanto el liderazgo transformacional como el liderazgo transaccional tienen impacto sobre los resultados de los estudiantes. Similarmente Gurr, Drysdale y Mulford [24], a partir de un estudio de casos realizado en Australia, sostienen que el liderazgo de la alta dirección es un proceso complejo que, no obstante, puede conducir a mejores resultados en los estudiantes. Complementariamente, en un estudio en 38 escuelas australianas se encuentran ciertos atributos, cualidades y prácticas de los directores de establecimientos que conducen a mayor enfoque en los estudiantes y en su aprendizaje [15]. En este mismo contexto, un estudio en 218 instituciones educativas de Canadá muestra que 
el liderazgo transformacional tiene un impacto sobre la eficacia colectiva e individual de los profesores, sobre su compromiso con la misión de la institución y sobre su compromiso y esfuerzo de enseñanza [65].

No obstante, otro conjunto de investigaciones apuntan en otra dirección o ponen en duda la validez de los resultados anteriores. Por ejemplo, en Holanda los resultados obtenidos para períodos distintos (1989 y 1993) son ambiguos en relación con el impacto del estilo de liderazgo y los resultados de los estudiantes [75]. La cuestión es que el liderazgo de la alta dirección se traduce en estrategias bien equilibradas que tienen impacto sobre la motivación de los profesores y de los estudiantes [4]. Sin embargo, la motivación no genera necesariamente mejoramiento en el desempeño [5]. Similarmente, en un estudio realizado en Bélgica se propone que el liderazgo no es capaz de afectar las prácticas de los colegios, ni sus resultados [51]. De modo equivalente, en una investigación desarrollada en Singapur se descubre que el liderazgo transformacional tiene efectos indirectos sobre los logros académicos de los estudiantes y el liderazgo transaccional prácticamente no tiene un efecto significativo sobre la calidad de los establecimientos educativos [41]. Consecuentemente, en un estudio en 655 colegios en Inglaterra se demuestra que el liderazgo tiene efecto sobre las prácticas docentes en el aula, pero no necesariamente sobre el desempeño de los estudiantes [43]. De igual modo, en un estudio de caso en una escuela con rendimiento excepcional en Inglaterra se encuentra que el liderazgo transformacional no se relaciona de manera positiva con el desempeño de los estudiantes [3].

El desafío intelectual y empírico consiste en integrar las dimensiones anteriores y los diferentes enfoques de análisis, considerando los estilos de liderazgo en el contexto de un proceso de interacción entre los líderes, los seguidores y el contexto situacional (siguiendo a Spillane, Halverson y Diamond [71]). De manera similar, resulta fundamental para el estado del arte identificar eventuales efectos directos del estilo de liderazgo sobre la calidad de los establecimientos educacionales, y fundamentalmente (siguiendo a [77, 49, 28, 74]) a evaluar las relaciones directas e indirectas de: estilos de liderazgo/gestión de recursos humanos/ resultados de los estudiantes en la P.S.U.

\section{Gestión de recursos humanos en organizaciones educativas}

La relación entre recursos de las escuelas y logros de los estudiantes no es clara. De hecho, las variables socioeconómicas e "inputs" de la familia parecen tener un impacto mayor en el logro de los estudiantes. A este respecto, un metaanálisis que considera 400 estudios de logro de los estudiantes no encuentra relaciones fuertes o consistentes entre los recursos de la escuela y los resultados de los estudiantes [30]. Por lo tanto, una buena política educativa no se trata simplemente de asignar mayores recursos a las escuelas o un monto mayor de asignación por estudiante para dotar de mayores recursos a las escuelas.

En efecto, una arista que puede ser incorporada dice relación con la gestión de los recursos humanos, los que van más allá de los recursos físicos y materiales, más allá de la infraestructura y del equipamiento. Ciertamente, algunos estudios importantes han reconocido la influencia que los profesores ejercen sobre los resultados de los estudiantes (por ejemplo [80, 78]). Sin embargo, la evidencia empírica no es suficientemente clara para probar qué características observables hacen efectivos a los profesores.

En este contexto, resulta importante revisar la literatura que relaciona la gestión de recursos humanos con los resultados de los estudiantes, dado que los resultados de los alumnos son la expresión más concreta de la calidad de la educación recibida. La cuestión que se pretende dilucidar es si existe una relación estructural entre gestión de recursos humanos y calidad de la educación. De manera más específica, se procura determinar si los procesos de selección que apuntan a discriminar según la preparación de los profesores, sus habilidades, sus certificaciones, su experiencia, unido a programas de perfeccionamiento, sistemas de retribución consistentes con el logro de objetivos medibles y verificables de calidad, y sistemas de evaluación grupal e individual, influyen o no sobre los resultados de los estudiantes.

Desde esta perspectiva [66], descubre que el logro de los estudiantes en matemática se puede asociar con un conjunto de variables que caben dentro de la gestión de recursos humanos. Estas variables son: la habilidad y conocimiento de los profesores, 
la motivación de los profesores y las condiciones de trabajo de los profesores. Sin embargo, el abandono de las funciones docentes de profesores de enseñanza básica y media para dedicarse a otros tipos de trabajos no es de extrañar en la evidencia internacional $\mathrm{y}$, precisamente, los profesores con mejores conocimientos y habilidades son aquellos más proclives a dejar la labor formadora (por ejemplo [23, 47]). Más aún, los profesores de las áreas de matemática y ciencias son aquellos que en mayor proporción abandonan la profesión docente (por ejemplo $[68,48]$.

Por lo tanto, las capacidades de los profesores y su flexibilidad para asumir otros tipos de labores, y las condiciones de trabajo, principalmente asociadas a la retribución y remuneraciones percibidas en los establecimientos educacionales, las condiciones globales del mercado del trabajo, entre otros aspectos, configuran los determinantes que generen movilidad y salida de los profesores del mercado docente o la propia movilidad dentro del mercado docente, en el cual los profesores buscan mejores condiciones laborales (por ejemplo [48, 62, 23]).

Por su parte, Lankford, Loeb y Wyckoff [42] demuestran que existe una relación altamente significativa entre la calidad global de los profesores y los resultados de los estudiantes. La calidad de los profesores se mide considerando una serie de variables que son posibles de tomar en cuenta en los procesos de selección. Dichos factores clave, entre otros similares, son: el grado académico, la experiencia profesional y la calidad de la institución. En la misma línea de desarrollo anterior, Kaplan y Owings [39] plantean que la alta dirección de las escuelas juega un rol fundamental al asegurar la calidad de los profesores y de la enseñanza, aspectos que son esenciales para el progreso y niveles de logro de los estudiantes.

Efectivamente, un estudio en escuelas de Texas prueba que la calidad de los profesores asociada a variables observables tales como nivel educacional y experiencia son determinantes esenciales de la calidad del establecimiento educativo [64].

En forma complementaria, se puede establecer que el nivel de perfeccionamiento de los profesores no es un tema menor. Por el contrario, un estudio de Darling-Hammond [14] concluye que políticas de inversión tendientes al perfeccionamiento de los profesores pueden relacionarse positivamente con los resultados que alcancen los estudiantes. En la investigación se demuestra cuantitativamente que existe una fuerte correlación entre la preparación formal de los profesores y los logros de los estudiantes en lenguaje y matemática.

Reichardt [63] sostiene que junto con los profesores, la formación de profesores que le compete a la educación superior, el reclutamiento y la selección de profesores, las políticas de mantención de docentes, y las políticas de perfeccionamiento de las habilidades y conocimiento de los profesores constituyen aspectos esenciales para lograr mayor calidad en la educación escolar. De modo similar, Allen [1] realiza un reporte editado por la Comisión de Educación de los Estados Unidos en el que, en varias ocasiones, alude a la importancia de los procesos de reclutamiento y selección de profesores, las condiciones de trabajo y otros aspectos similares asociados a la gestión de recursos humanos.

Tal y como lo plantea Hanushek [31], la calidad de la educación no se resuelve simplemente con mayores recursos o asignaciones presupuestarias. Los profesores juegan un papel fundamental y la medición de desempeño y la asignación de incentivos asociados a la performance de los profesores son aspectos cruciales para impactar en la calidad de la educación impartida. En esta misma línea de dirección, Ballou y Podgursky [2] argumentan en favor de la rendición de cuenta por parte de los profesores y los establecimientos educativos y sugieren la pertinencia de emplear herramientas de gestión que asocien las remuneraciones de los profesores a su desempeño.

En su trabajo de tesis doctoral Kimball [40] descubre que hay una relación positiva entre las remuneraciones de los profesores, su retención o mantención en los establecimientos educacionales y los resultados de los estudiantes. Por lo tanto, la efectividad de relacionar incentivos con resultados de los estudiantes no sólo conduce a una mayor calidad. Por lo demás, la evaluación no tiene que ser de naturaleza punitiva. Por el contrario, los procesos de carácter más participativos y colaborativos pueden mejorar la eficacia de los profesores [10]. 


\section{CONCLUSIONES}

Los diversos estudios que se han realizado en la última década acerca del sistema educacional chileno evidencian una conclusión preocupante: la calidad de la educación no ha mejorado sustantivamente. Ello, a pesar de los mayores niveles de inversión del Estado en la década de los 90 y de las diversas iniciativas implementadas en el marco de la reforma educativa. Estas investigaciones relevan la importancia de la condición social y económica de los estudiantes como el factor más determinante de los resultados del Sistema de Medición de la Calidad de la Educación (SIMCE), así como de la prueba de selección universitaria (P.S.U.).

En este contexto, es que resulta fundamental complementar la mirada de análisis incorporando la relevancia de la administración de los establecimientos educacionales, desde la perspectiva de la gestión de los recursos humanos y de los estilos de liderazgo como variables moderadoras de la calidad de la educación básica chilena.

En consecuencia, esta reflexión pone de manifiesto que la Ingeniería Industrial y la Gestión de Organizaciones pueden tener una significancia mayor al considerar los resultados del aprendizaje de los estudiantes, ya que tanto los estilos de liderazgo como los procesos de gestión de los recursos humanos constituyen variables que inciden decisivamente en la calidad de los procesos de enseñanza y en los resultados de los estudiantes.

\section{AGRADECIMIENTOS}

Los autores agradecen a la Comisión Nacional de Ciencia y Tecnología de Chile por el patrocinio a este trabajo a través del proyecto FONDECYT 1090298.

\section{REFERENCIAS}

[1] M. Allen. "Eight questions on teacher recruitment and retention: what does the research say?". Education commission of the States. 2005.

[2] D. Ballou and M. Podgursky. "Teacher Recruitment and Retention in Public and Private Schools". Journal of Policy Analysis and Management. Vol. 17, Issue 3, pp. 393417. 1998.
[3] B. Barker. "The leadership paradox: Can school leaders transform student outcomes?". Education Management. Vol. 18, Issue 1, pp. 21-43. 2007.

[4] B. Barker. "Do Leaders Matter?'. Educational Review. Vol. 53, Issue 1, pp. 65-76. 2001.

[5] B. Barker. "Transforming schools: illusion or reality?". School Leadership and Management. Vol. $25 \mathrm{~N}^{\circ}$ 2, pp. 99-116. 2005.

[6] J.C. Barlett. "Principal leadership practices: a correlation study of specific instructional leadership practices and student achievement on the Tennessee gateway tests". Disertación ed. D, Liberty University, p. 150. Virginia, Estados Unidos. 2008.

[7] R. Barro. "Economic growth in a cross-section of countries". Quarterly Journal of Economics. Vol. 106, Issue 2, pp. 407-443. 1991.

[8] A. Bassanini and S. Scarpetta. "Does human capital matter for growth in OECD countries? A Pooled Mean-Group Approach". Economics Letters. Vol. 74, Issue 3, pp. 399-405. February, 2002.

[9] J. Benhabib and M. Spiegel. "The role of human capital in economic development: evidence from aggregate cross-country data". Journal of Monetary Economics. Vol. 34, Issue 2, pp. 143-173. 1994.

[10] S. Black. "How Teachers Are Reshaping Evaluation Procedures". Educational Leadership. Vol. $51 \mathrm{~N}^{\circ}$ 2, pp. 38-42. October, 1993.

[11] M. Carpenter and J. Fredrickson. "Top Management Teams, Global Strategy Posture, and the Moderating Role of Uncertainty". The Academy of Management Journal. Vol. 44 $\mathrm{N}^{\mathrm{o}}$ 3, pp. 533-546. June, 2001.

[12] E. Carroll and M. Hannan. "The Demography of Corporations and Industries". Long Range Planning. Vol. 33, p. 608. 2000.

[13] J. Cooper. "High-performing high schools: patterns of success". National Association of Secondary School Principals Bulletin. Vol. 89 $\mathrm{N}^{\mathrm{o}} 645$, pp. 2-23. 2005.

[14] L. Darling-Hammond. "Teacher quality and student achievement: a review of state policy evidence". Center for the study of teaching and policy. University of Washington, pp. 1-48. 1999.

[15] S. Dinham. "Principal Leadership for outstanding educational outcomes". Journal of Educational Administration. Vol. 43, Issue 4, pp. 338-356. 2005. 
[16] S. Donoso e G. Hawes. "Eficiencia escolar y diferencias socioeconómicas: a propósito de los resultados de las pruebas de medición de la calidad de la educación en Chile". Educação e Pesquisa. Vol. 28 Nº 2, pp. 25-39. São Paulo, Brasil. 2002.

[17] S. Dowrick. "The Contribution of Innovation and Education to Economic Growth". Melbourne Institute Economic and Social Outlook. Conference, pp. 1-26. April 4-5, 2002.

[18] E. Duflo. "Schooling and labor market consequences of school construction in Indonesia: evidence from an unusual policy experiment". American Economic Review. Vol. 91, Issue 4, pp. 795-814. 2001.

[19] G. Evkall and L. Ryhammar. "Leadership style, social climate and organizational outcomes: A study of Swedish University College". Creativity and Innovation Management. Vol. 7, Issue 3, pp. 126-130. September, 1998.

[20] D. Frantzen. "A cross-country analysis r\&d, human capital and international technology spillovers". Scandinavian Journal of Economics. Vol. 102, Issue 1, pp. 57-75. 2000.

[21] D. Goleman. "Leadership that gets results". Harvard Business Review. Vol. 78, Issue 2, pp. 78-90. 2000.

[22] S. Gregory. "Leadership Styles and Characteristics of Effective Principals in High-Poverty Public Schools". Annual Meeting of the American Educational Research Association, pp. 1-14. 2003.

[23] C. Guarino, L. Santibáñez and G. Daley. "Teacher Recruitment and Retention: A Review of the Recent Empirical Literature". Review of Educational Research. Vol. 76, Issue 2, pp. 173-208. Summer 2006.

[24] D. Gurr, L. Drysdale and B. Mulford. "Successful principal leadership: Australian case studies". Journal of Educational Administration. Vol. $43 \mathrm{~N}^{\circ}$ 6, pp. 539-551. 2005.

[25] J. Haleblian and S. Finkelstein. “Top management team size, CEO dominance and firm performance". Academy of Management Journal. Vol. $36 \mathrm{~N}^{\mathrm{o}}$ 4, pp. 844-863. August, 1993.

[26] P. Hallinger and J. Murphy. "Assessing the instructional management behavior of principals". Elementary School Journal. Vol. 86, Issue 2, pp. 217-247. 1985.
[27] D. Hambrick and P. Mason. "Upper echelons: The organization as a reflection of its top managers". Academy of Management Review. Vol. 9, Issue 2, pp. 193-206. 1984.

[28] P. Hammond and T. Yeshanew. "The impact of feedback on school performance". Educational Studies. Vol. 33, Issue 2, pp. 99-113. 2007.

[29] M. Hannan and J. Freeman. "The population ecology of organizations". American Journal of Sociology. Vol. 82, Issue 5, pp. 929-964. 1977.

[30] E. Hanushek. "Assessing the Effects of School Resources on Student Performance: An Update". Educational Evaluation and Policy Analysis. Vol. 19, Issue 2, pp. 141-164. Summer 1997.

[31] E. Hanushek. "Publicly provided education. Cambridge". NBER Working Paper Series. Vol. 8799. 2002.

[32] E. Hanushek. "Interpreting recent research on schooling in developing countries". World Bank Research Observer. Vol. 10, Issue 2, pp. 227-246. 1995.

[33] A. Harris. "Effective Leadership in Schools Facing Challenging Contexts". School Leadership and Management. Vol. $22 \mathrm{~N}^{\circ} 1$, pp. 15-26. February, 2002.

[34] R. Hatcher. "The distribution of leadership and power in schools". British Journal of Sociology of Education. Vol. $26 \mathrm{~N}^{\mathrm{o}} 1$, pp. 253267. April, 2005.

[35] R. Hofmann, W. Hofmann and H. Gundelond. "The effectiveness of cohesive schools". International Journal of Leadership in Education. Vol. 4, Issue 2, pp. 115-135. 2001.

[36] H.-C. Hsiao, M.-N. Chen and H.-S. Yang. "Leadership of vocational high school principals in curriculum reform: A case study in Taiwan". International Journal of Educational Development. Vol. 28, Issue 6, pp. 669-686. November, 2008.

[37] S. Hui and H. Cheung. "A Re-examination of Leadership Style for Hong Kong SchoolBased Management Schools". Asia Pacific Journal of Education. Vol. $26 \mathrm{~N}^{\mathrm{o}}$ 2, pp. 173187. November, 2006.

[38] S. Kahai and J. Sosik. "Effects of Leadership Style and Followers' Cultural Orientation on Performance in Group and Individual Task Conditions". Personell Psychology. Vol. 50, Issue 1, pp. 121-147. March, 1997.

[39] L. Kaplan and W. Owings. "Teacher Quality and Student Achievement: Recommendations 
for Principals". NASSP Bulletin. Vol. 85 $\mathrm{N}^{\circ}$ 628, pp. 64-73. 2001.

[40] S.M. Kimball, B. White, A.T. Milanowski and G. Borman. "Examining the Relationship between Teacher Evaluation and Student Assessment Results in Washoe County". Peabody Journal of Education. Vol. 79, Issue 4, pp. 54-78. 2004. DOI: 10.1207/ s15327930pje7904_4

[41] H. Koh, R. Steers and J. Terborg. "The Effects of Transformational Leadership on Teacher Attitudes and Student Performance in Singapore". Journal of Organizational Behavior. Vol. 16, Issue 4, pp. 319-333. July, 1995.

[42] H. Lankford, S. Loeb and J. Wyckoff. "Teacher sorting and the plight of urban schools: A descriptive analysis". Educational Evaluation and Policy Analysis. Vol. $24 \mathrm{~N}^{\mathrm{o}}$ 1, pp. 37-62. 2002.

[43] K. Leithwood and D. Jantzi. "Transformational school leadership for large-scale reform: Effects on students, teachers, and their classroom practices". School Effectiveness and School Improvement. Vol. $17 \mathrm{~N}^{\mathrm{o}} 2$, pp. 201-227. June, 2006.

[44] M. Lockheed and A. Verspoor. "Improving Primary Education in Developing Countries". Oxford University Press. New York, United States. 1991.

[45] R. Lucas. "On the Mechanics of Economic Development". Journal of Monetary Economics. Vol. 22, pp. 3-42. 1988.

[46] N. Mankiw, D. Romer and D. Weil. "A contribution to the empirics of economic growth". Quarterly Journal of Economics. Vol. 107, Issue 2, pp. 407-437. 1992.

[47] R.J. Murnane, J.D. Singer and J.B. Willett. "The influences of salaries and opportunity costs on teachers' career choices: Evidence from North Carolina". Harvard Educational Review. Vol. 59, Issue 3, pp. 325-346. 1989.

[48] R. Murnane, J. Singer, J. Willett, J. Kemple and R. Olsen. "Who will teach? Policies that matter". Harvard University Press. Cambridge, MA, United States. 1991.

[49] S. Nguni, P. Sleegers and E. Denessen. "Transformational and transactional leadership effects on teachers' job satisfaction, organizational commitment, and organizational citizenship behavior in primary schools: The Tanzanian case". School
Effectiveness and School Improvement. Vol. $17 \mathrm{~N}^{\mathrm{o}}$ 2, pp. 145-177. June, 2006.

[50] E. Ogbonna and L. Harris. "Leadership Style, Organizational Culture and Performance: Empirical Evidence from U.K. Companies". International Journal of Human Resources Management. Vol. $11 \mathrm{~N}^{\circ}$ 4, pp. 766-788. August, 2000.

[51] M. Opdenakker and J. van Damme. "Do school context, student composition and school leadership affect school practice and outcomes in secondary education?". British Educational Research Journal. Vol. $33 \mathrm{~N}^{\circ} 2$, pp. 179-206. April, 2007.

[52] L. Pedraja y E. Rodríguez. "Efectos del estilo de liderazgo sobre la eficacia de las organizaciones públicas". Revista Facultad de Ingeniería-Universidad de Tarapacá. Vol. 12 $\mathrm{N}^{\circ}$ 2, pp. 63-73. Arica, Chile. 2004.

[53] L. Pedraja-Rejas y E. Rodríguez-Ponce. "Importancia de los estilos de liderazgo sobre la eficacia: un estudio comparativo entre grandes y pequeñas y medianas empresas privadas". Revista de Ciencias Sociales (RCS) Vol. XIV No 1, pp. 20-29. Enero-abril 2008.

[54] L. Pedraja-Rejas, E. Rodríguez-Ponce y J. Rodríguez-Ponce. "Sociedad del conocimiento y dirección estratégica: una propuesta integradora”. Interciencia, Journal of Science and Technology of the Americas. Vol. $31 \mathrm{~N}^{\circ}$ 8, pp. 570-576. Agosto, 2006.

[55] L. Pedraja-Rejas, E. Rodríguez-Ponce y J. Rodríguez-Ponce. "Liderazgo y decisiones estratégicas: una perspectiva integradora". Interciencia, Journal of Science and Technology of the Americas. Vol. $31 \mathrm{~N}^{\mathrm{o}} 8$, pp. 577-582. August, 2006.

[56] L. Pedraja-Rejas, E. Rodríguez-Ponce y J. Rodríguez-Ponce. "Estilos de liderazgo y eficacia: un estudio en pequeñas empresas en Chile". Interciencia, Journal of Science and Technology of the Americas. Vol. 31 $\mathrm{N}^{\mathrm{o}}$ 7, pp. 500-504. July, 2006.

[57] L. Pedraja-Rejas y E. Rodríguez-Ponce. "Estudio comparativo de la Influencia del estilo de liderazgo y la congruencia de valores en la eficacia de empresas privadas e Instituciones Públicas". Interciencia, Journal of Science and Technology of the Americas, Vol. $33 \mathrm{~N}^{\mathrm{o}}$ 1, pp. 8-13. January, 2008.

[58] L. Pelled, K. Eisenhardt and K. Xin. "Exploring the Black Box: An Analysis 
of Work Group Diversity, Conflict, and Performance". Administrative Science Quarterly. Vol. 44 N$^{\circ}$ 1, pp. 1-28. March, 1999.

[59] G. Psacharopoulos. "Returns to Education: A Further International Update and Implications". Journal of Human Resources. Vol. 20, Issue 4, pp. 583-604. 1985.

[60] G. Psacharopoulos. "Returns to Investment in Education: A Global Update". World Development. Vol. 22, Issue 9, pp. 13251344. September, 1994.

[61] S. Rahman. "Total Quality Management Practices and Business Outcome: Evidence from Small and Medium Enterprises in Western Australia”. Total Quality Management. Vol. 12, Issue 2, pp. 201-210. 2001.

[62] D.I. Rees. "Grievance Procedure Strength and Teacher Quits". Industrial and Labor Relations Review. Vol. 45, Issue 1, pp. 31-43. 1991.

[63] R. Reichardt. "Toward A Comprehensive Approach to Teacher Quality". Mid-Continent Research for Education and Learning, pp. 1-13. 2001.

[64] S. Rivkin, E. Hanushek and J. Kain. "Teachers, Schools, and Academic Achievement". Econometrica. Vol. $73 \mathrm{~N}^{\circ}$ 2, pp. 417-458. March, 2005.

[65] J. Ross and P. Gray. "Transformational leadership and teacher commitment to organizational values: The mediating effects of collective teacher efficacy". School Effectiveness and School Improvement. Vol. 17 No 2, pp. 179-199. June, 2006.

[66] B. Rowan, F. Chiang and R. Miller. "Using Research on Employees' Performance to Study the Effects of Teachers on Students' Achievement". Sociology of Education. Vol. $70 \mathrm{~N}^{\circ}$ 4, pp. 256-284. 1997.

[67] B. Shamir, R. House and M. Arthur. "The motivational effects of charismatic leadership: A self-concept based theory". Organization Science. Vol. 4, pp. 577-594. 1993.

[68] H. Shin. "Estimating future teacher supply: Any policy implications for educational reform?" International Journal of Educational Reform. Vol. 4, Issue 4, pp. 422-433. 1995.

[69] H. Silins. "The Relationship between Transformational and Transactional Leadership and School Improvement Outcomes". School Effectiveness and School Improvement. Vol. 5, pp. 272-298. 1994.
[70] T. Simonds, L. Pelled and K. Smith. "Making use of difference: diversity, debate, and decision comprehensiveness in top management teams". Academy of Management Journal. Vol. $42 \mathrm{~N}^{\mathrm{o}}$ 6, pp. 662673. 1999.

[71] J. Spillane, R. Halverson and J. Diamond. "Towards a theory of leadership practice: a distributed perspective". Journal of Curriculum Studies. Vol. $36 \mathrm{~N}^{\circ}$ 1, pp. 3-34. 2004.

[72] J. Spillane, R. Halverson and J. Diamond. "Investigating school leadership practice: a distributed perspective". Educational Researcher. Vol. 30, Issue 3, pp. 23-28. April, 2001.

[73] J. Stiglitz. "La Economía del sector público". Tercera edición. Antonio Bosch. Madrid, España. 2002.

[74] S. Thomas. "Leading for quality: questions about quality and leadership in Australia". Journal of Education Policy. Vol. 23, Issue 3, pp. 323-334. 2008.

[75] W. van de Grift and A. Houtveen. "Educational leadership and pupil achievement in primary education". School Effectiveness and School Improvement. Vol. 10, Issue 4, pp. 373-389. 1999.

[76] D. Waldman, G. Ramírez, R. House and P. Puranam. "Does leadership matter, CEO leadership attributes and profitability under conditions of perceived environmental uncertainty". Academy of Management Journal. Vol. $44 \mathrm{~N}^{\circ}$ 1, pp. 134-143. 2001.

[77] M. Wallace. "Modelling Distributed Leadership and Management Effectiveness: Primary School Senior Management Teams in England and Wales". School Effectiveness and School Improvement. Vol. $13 \mathrm{~N}^{\mathrm{o}} 2$, pp. 163-186. 2002.

[78] S. Wright, S. Horn and W. Sanders. "Teacher and classroom context effects on student achievement: implications for teacher evaluation". Journal of Personnel Evaluation in Education. Vol. 11, pp. 57-67. 1997.

[79] G. Yukl. "Leadership in Organizations". 5th Edition. Upper Saddle River, NJ: Prentice Hall. 2002.

[80] B. Rowan, R. Correnti and R. Miller. "What large-scale survey research tells us about teacher effects on student achievement: insights from the 'Prospects' study of elementary schools". Teachers College Record. Vol. 104, Issue 8, pp. 1525-1567. 2002. 